\title{
Wide Range Fiber Displacement Sensor Based on Bending Loss
}

\author{
Jinlei Zhao, ${ }^{1,2}$ Tengfei Bao, ${ }^{1,2}$ and Tribikram Kundu ${ }^{3}$ \\ ${ }^{1}$ State Key Laboratory of Hydrology-Water Resources and Hydraulic Engineering, Hohai University, Nanjing 210098, China \\ ${ }^{2}$ College of Water-Conservancy and Hydropower, Hohai University, Nanjing 210098, China \\ ${ }^{3}$ Department of Civil Engineering and Engineering Mechanics, University of Arizona, Tucson, AZ 85721, USA
}

Correspondence should be addressed to Tengfei Bao; baotf@hhu.edu.cn

Received 23 August 2015; Revised 11 December 2015; Accepted 11 January 2016

Academic Editor: Lei Su

Copyright (C) 2016 Jinlei Zhao et al. This is an open access article distributed under the Creative Commons Attribution License, which permits unrestricted use, distribution, and reproduction in any medium, provided the original work is properly cited.

\begin{abstract}
A wide range fiber optic sensor system for displacement and crack monitoring is developed. In the proposed fiber optic sensor system, a number of fiber loops are formed from a single fiber and each fiber loop is used as a crack or displacement sensor. The feasibility and the dynamic range of the fiber sensor developed in this manner are investigated experimentally. Both glass fibers and plastic fibers are used in the experiments. Experimental results show that the new fiber optic sensor has a wide range (maximum range is $88 \mathrm{~mm}$ ) and this sensor also has a high sensitivity for displacement and crack monitoring when an appropriate diameter of the fiber loop is selected as the sensor. Moreover, the proposed method is very simple and has low cost, so in situ application potential of the proposed sensor is high.
\end{abstract}

\section{Introduction}

In the past forty years, fiber optic sensors have been used in different fields for various purposes because of their advantages, such as small size, lightweight, immunity to electromagnetic interference, and high sensitivity. A number of fiber optic sensors have been developed for displacement or crack monitoring. Rossi and Le Maou [1] first embedded optical fibers in concrete to detect cracks from the complete disappearance of signal in the fibers caused by the cracks. Ansari and Navalurkar [2] embedded fiber loops in cementitious composites to measure crack tip opening displacements based on the macrobend effect of fibers. Ohno et al. [3] described the principle of optical fiber strain sensors using a Brillouin optical-domain reflectometer (BOTDR). Leung et al. $[4,5]$ developed a "zig-zag" crack fiber sensor based on optical time domain reflectometry (OTDR). The "zig-zag" sensor performs well for small crack monitoring and a priori knowledge of crack location is not required. Yuan et al. [6] developed an optical fiber ultrasonic sensor based on Fizeau interferometer to monitor the existence and development of microcracks inside concrete structures. Zhang and Ansari [7] developed a fiber optic laser speckle-intensity crack sensor which is effective for monitoring microcracks. The fiber Bragg grating has been also used widely as an optical sensor for its high strain resolution.

In recent years, plastic optical fibers (POFs) are receiving the interest of researchers due to their high resistance to strain, low cost, and ease of handling. The POF is the only optical fiber that can be installed without any special tool. Kuang et al. [8] attached POFs on concrete beams to detect crack initiation and monitor postcrack vertical deflection. Later, Kuang et al. $[9,10]$ developed an extrinsic polymerbased fiber optic sensor for structural health monitoring. Liehr et al. [11-13] described the distributed strain measurement capability of POFs for structural health monitoring using OTDR technique in their papers.

A single fiber optic sensor can monitor a large number of cracks. This distributed sensing capability of a fiber optic sensor makes it an attractive sensing tool. However, traditional glass fibers are brittle and easy to break. As a result, the measurement range of most glass fibers for crack sensing is small. The glass fiber breaks when a large displacement or crack occurs at one location, and then the distributed sensing capability of the fiber is destroyed. POFs appear to be a good substitute for traditional glass fibers. However, high optical attenuation of POFs limits the sensing distance. Husdi et al. [14] have reported in their paper that the sensing distance 


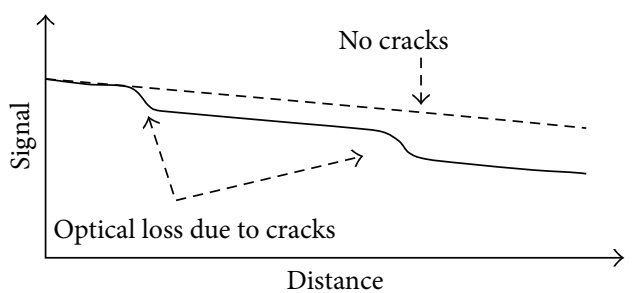

(a)

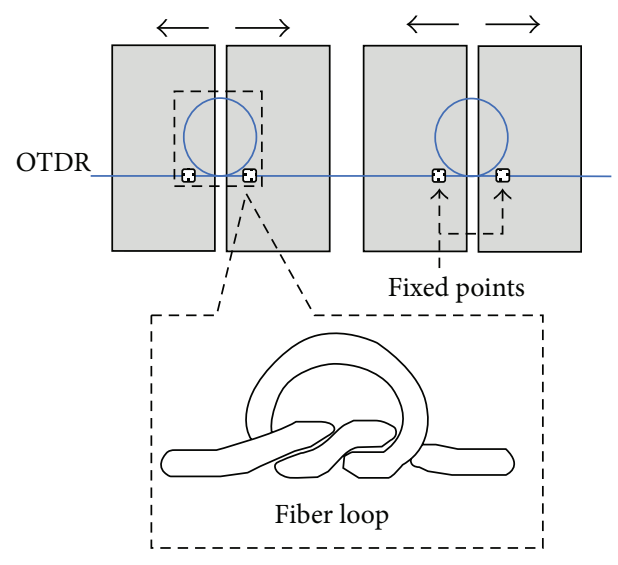

(b)

Figure 1: The crack sensing concept.

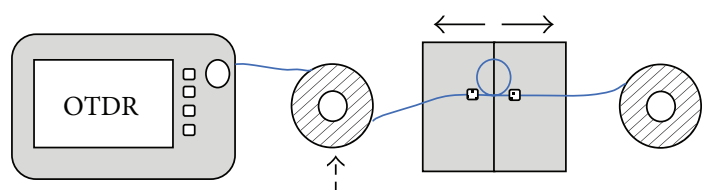

A spool of silica fiber

FIgURE 2: Schematic diagram of experimental setup for glass fibers.

of POFs can reach about $120 \mathrm{~m}$ using OTDR technique, while the measurement distance of glass fibers can exceed $10 \mathrm{~km}$ with OTDR technique. Therefore, it is still necessary to find a method to improve the measurement range of glass optical fibers for crack and deformation monitoring for their capability of long distance sensing.

In this paper, a fiber sensor system with a large measurement range for displacement and crack monitoring is developed. For this sensor system, a large number of fiber loops are formed from a single fiber and each fiber loop can be used as a sensor, so a priori knowledge of crack location is not required. In practice, several fibers can be employed to increase the sensing area. Tests are conducted both on glass fibers and plastic fibers as described in the following sections.

\section{Sensing Principle}

As shown in Figure 1, two loops are formed from a single fiber and each loop is attached to the surface of a structure only at two "fixed points" (shown by two small squares in Figure 1). When a crack occurs, the two fixed points on two sides of the crack move apart. Therefore, the radius of the fiber loop changes is reduced gradually with the development of the crack. At the same time, optical loss is induced at this location and the optical loss increases with the development of the crack, which can be monitored by an OTDR or a power meter. For the fiber sensors based on OTDR technique, the loss at one location is independent of that in the other part of the fiber [5]. Therefore, a single fiber with a large number of loops can monitor a large number of cracks.

The sensing principle of the fiber sensor proposed here can be explained by the macrobend effect of fibers. When a fiber is bent at a location, optical loss is induced at that location. The mechanism behind the bending loss is explained below. The refractive index of the cladding is lower than that of the fiber core. For the straight position of the fiber, a light that is launched into the core at a low angle measured from its axis undergoes total internal reflection between the core and cladding interface. However, when the fiber is bent some of the light escapes from the core and the cladding due to the change of the incidence angle. This loss is given by the following expression [15]:

$$
\text { Loss }=\frac{T}{2 \sqrt{R}} \exp \left[2 W a-\frac{2 W^{3}}{3 \beta^{2}} R\right],
$$

where $T=2 a k^{2} / e \sqrt{\pi W} V^{2}, U^{2}=n_{1}^{2} k_{0}^{2}-\beta^{2}, W^{2}=\beta^{2}-n_{2}^{2} k_{0}^{2}$, $V^{2}=a^{2} k_{0}^{2}\left(n_{1}^{2}-n_{2}^{2}\right)$, and $a$ is the radius of the fiber core. $R$ is the radius of the bend. $k=2 \pi / \lambda, k_{0}=2 \pi / \lambda_{0}$. T is the total birefringence effect of the fiber. $\beta$ is the phase constant. $\nu$ is Poisson's ratio. $n_{1}$ and $n_{2}$ are the refractive indices of the fiber core and cladding, respectively.

Theoretically, there is a critical bend radius for fibers. When the radius of the bend is bigger than the critical bend radius, there is no optical loss. On the other hand, when the radius of the bend is smaller than the critical bend radius, the optical loss is induced at the bend location. The critical bend radius is important for the sensor proposed here. The critical bend radius for both glass fibers and POFs are investigated experimentally in the following sections.

\section{Experimental Setup}

Wan and Leung [5] have shown that the optical loss at one location of a fiber does not influence that at another location using OTDR. Therefore, the optical loss monitored by an OTDR at one fiber loop does not influence that at other loops in the proposed fiber sensor system. In the test, one fiber loop was bent for a sensor to investigate the capability of the proposed bending-based sensor in crack monitoring.

3.1. Test Setup for Glass Fiber. As shown in Figure 2, the experimental setup for glass fibers consists of an OTDR (MW9076B7), two spools of $500 \mathrm{~m}$ glass fiber (Corning SM$28 \mathrm{e})$, and two epoxy blocks used as a crack simulator. In the test, the light of $1310 \mathrm{~nm}$ wavelength is incident into the fiber using the OTDR. The two fiber spools in the setup are used to avoid the possibility of the weak backscattered signals at the fiber loops being overwhelmed in loss monitoring. The crack simulator can be opened gradually and the optical loss, the 
TABLE 1: Specification of the fibers used in tests.

\begin{tabular}{lccc}
\hline Item & Unit & SMF-28e & SK10/SK20 \\
\hline$\emptyset$ core & $\mu \mathrm{m}$ & 10 & $250 / 500$ \\
$\emptyset$ cladding & $\mu \mathrm{m}$ & 125 & $250 / 500$ \\
Max transmission loss & $\mathrm{dB} / \mathrm{km}$ & $0.35($ at $1310 \mathrm{~nm})$ & $300 / 180($ at $650 \mathrm{~nm})$ \\
Max operating temperature & ${ }^{\circ} \mathrm{C}$ & $85 \mathrm{Max}$ & $70 \mathrm{Max}$ \\
\hline
\end{tabular}

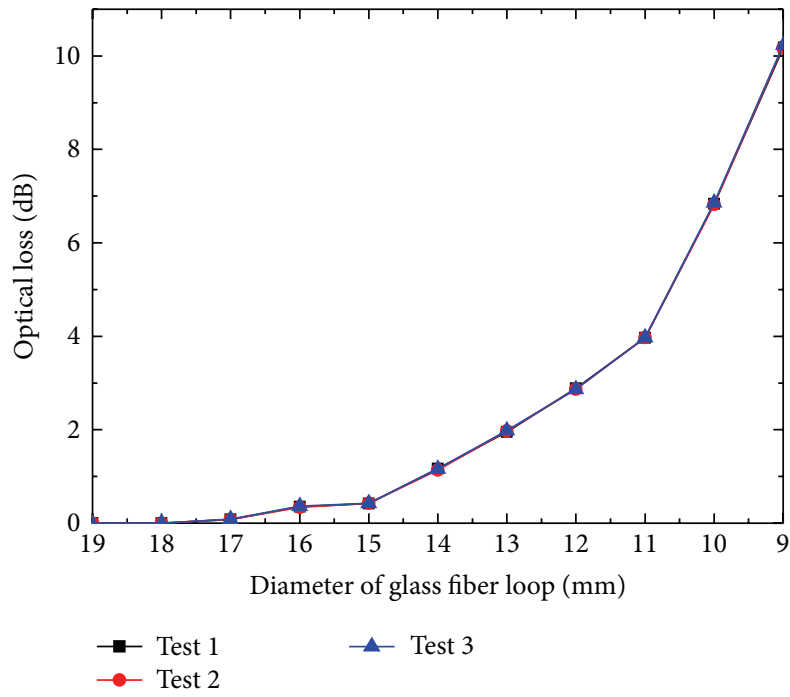

FIGURE 3: Relationships between optical loss and glass fiber loop diameter.

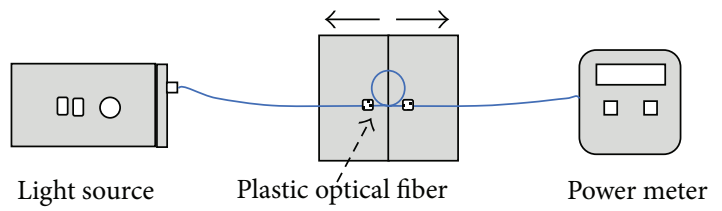

FIgURE 4: Schematic diagram of experimental setup for POFs.

diameter of the fiber loop, and the crack opening are recorded simultaneously. The specific fiber diameter inducing bending loss is determined by the fiber core diameter, fiber material, experimental environment, and so forth. In this study, the specific fiber diameter was tested by experimental methods. Three group tests were conducted at the same experimental environment for the glass fiber used in this study. From the test results shown in Figure 3, when the diameter of the glass fiber loop is about $17 \mathrm{~mm}$, the OTDR can monitor optical loss at the location of the fiber loop.

3.2. Test Setup for POFs. The experimental setup for POFs is shown in Figure 4. The setup is composed of a light source, POFs, a power meter, and two epoxy blocks used as a crack simulator. The light with $650 \mathrm{~nm}$ wavelength is generated by a light source and is propagated through the POF. Two kinds of POFs are used in the test, ESKA SK10 and SK20, made by Mitsubishi Rayon. The parameters of the POFs and the glass fibers used are shown in Table 1. Compared to the glass

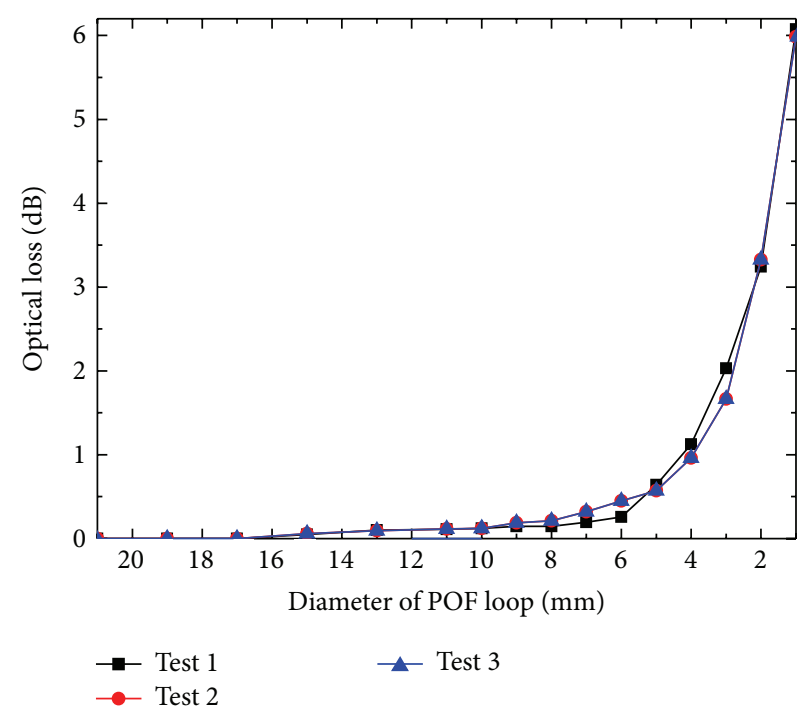

FIGURE 5: Relationships between optical loss and POF loop diameter (SK10).

fiber, the POFs have a bigger core diameter and a bigger transmission loss. Similar to the glass fiber, the specific fiber diameters inducing bending loss in this study were tested by experimental methods. Test results in Figures 5 and 6 showed that the optical loss of the two kinds of POFs shows a clear increasing trend with the decrease of the fiber loop diameter and bending loss occurs when the diameter of the POF loop is about $17 \mathrm{~mm}$ and $29 \mathrm{~mm}$ for SK10 and SK20, respectively.

\section{Experimental Results and Analysis}

For crack monitoring using fiber sensors, the relationship between the optical loss and crack openings is very important. For this reason, the optical loss versus the crack opening relation is studied in this paper.

4.1. The Experimental Results with the Glass Fibers. The optical loss of the glass fibers increases gradually with the increase of the crack opening and the measurement range can reach $26 \mathrm{~mm}$, as shown in Figure 7. The loss increases slowly with the crack opening initially, but later it increases at a much higher rate. From these results, one can see that the optical loss rate increases noticeably when the crack opening exceeds 10 or $15 \mathrm{~mm}$ and the diameter of the fiber loop is less than 14 or $12.5 \mathrm{~mm}$. In the test, the OTDR exceeds its dynamic range when the diameter is reduced to $9 \mathrm{~mm}$ due to high 


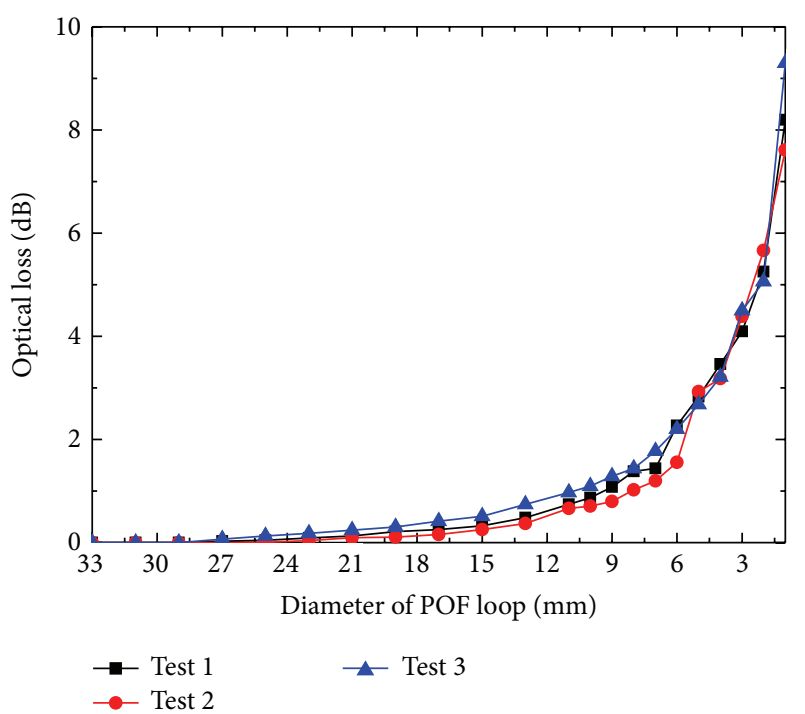

FIGURE 6: Relationships between optical loss and POF loop diameter (SK20).

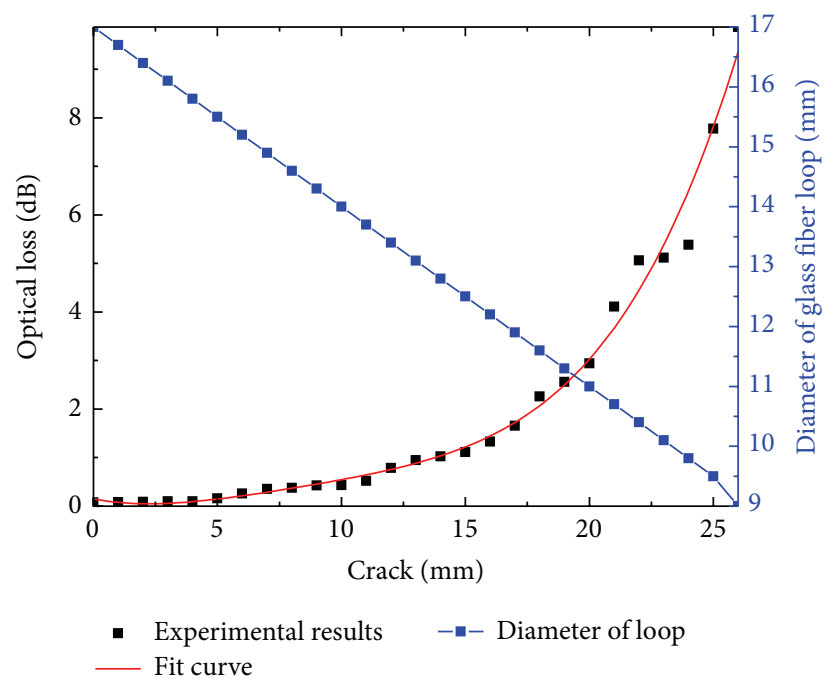

FIGURE 7: Dependence of optical loss and glass fiber loop diameter on crack opening.

optical loss. From Figure 7 it can be seen that in the highloss region the fiber is more sensitive to the crack opening displacement, and in the low-loss region it is less sensitive to this displacement. Therefore, it is desirable to have the fiber diameter smaller than $14 \mathrm{~mm}$ to maintain high sensitivity of the sensor.

4.2. The Experimental Results with the POFs. The experimental results with the POFs are shown in Figures 8 and 9. Similar to the experimental results for the glass fiber discussed above, the optical loss of the two kinds of POFs shows a clear increasing trend with the development of the crack. The maximum measurement ranges of the SK10 and SK20 are $50 \mathrm{~mm}$ and $88 \mathrm{~mm}$, respectively. Compared to SK10, the optical loss measured in SK20 at its maximum measurement

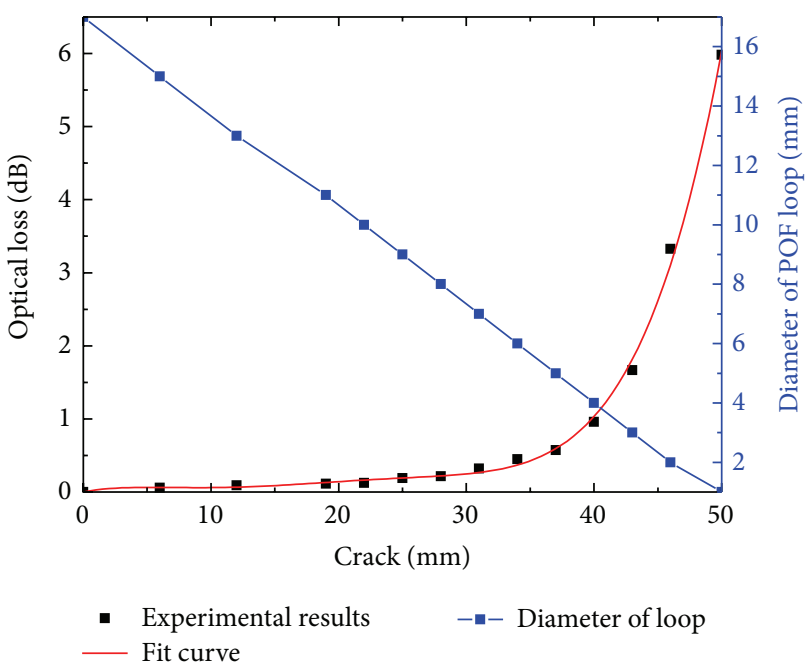

FIgure 8: Dependence of optical loss and POF fiber loop diameter on crack opening (SK10).

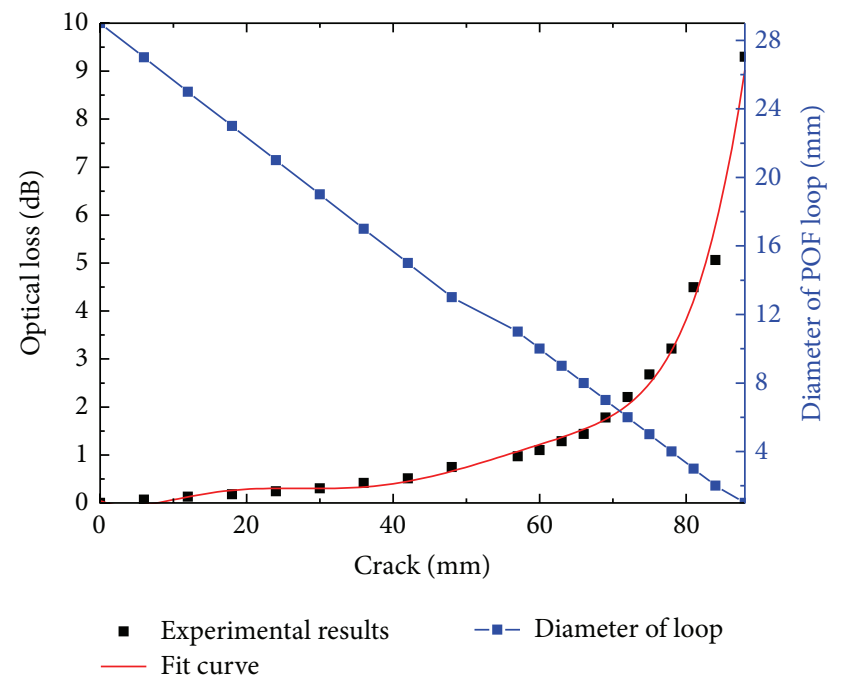

FIgURE 9: Dependence of optical loss and POF fiber loop diameter on crack opening (SK20).

range is higher as shown in the results because of its larger core diameter. Moreover, the sensitivity of SK20 for crack monitoring is higher than that of the SK10, especially when the crack opening is small. Therefore, the POFs with a bigger core diameter perform better for crack monitoring. Similar to the sensor made of glass fibers, it is important to choose an appropriate initial diameter for the sensor in practice due to different rates of optical loss at different phases of crack opening.

\section{Comparison with Previous Works}

In the previous work by Leung et al. $[4,5]$, they also developed a crack sensor based on glass fibers. The crack sensor performs well for small crack (crack openings below $2 \mathrm{~mm}$ ) monitoring. The glass fiber sensor presented in the present 
paper is quite different from the sensor proposed by Wan and Leung. Our sensor mainly focuses on the monitoring of large cracks (crack openings between $0 \mathrm{~mm}$ and $26 \mathrm{~mm}$ ). Since the two sensors are both made of single mode glass fibers, it is possible to combine the two sensors in field applications.

Kuang et al. [8] applied POFs on crack detection and vertical deflection monitoring in concrete beams. They created structural imperfections on POFs by abrading the surfaces of the POFs. Therefore, their POF sensor has a high sensibility due to the structural imperfections. However, the high sensibility will cost its dynamic range inevitably. Our POF sensor made of POFs without structural imperfections has a wide range in crack monitoring. It is also possible to combine the two POF sensors in field applications.

\section{Conclusions}

A very simple, distributed fiber optic sensor system for displacement and crack monitoring is proposed. The sensor system is built by bending the fiber and forming several loops from a single fiber. Then the same number of cracks as the number of loops can be monitored by the single fiber. The fiber sensors have a large measurement range $(26 \mathrm{~mm}$ for glass fiber SMF-28e and $88 \mathrm{~mm}$ for POF SK20). This investigation shows that the POFs have a high potential for displacement or crack monitoring because of its low cost and relative ease in handling.

This bending-based fiber sensor presented in this paper can be combined with other high precision fiber sensors. Future work on this fiber sensor will involve investigating its monitoring capability in real structures.

\section{Conflict of Interests}

The authors declare that there is no conflict of interests regarding the publication of this paper.

\section{Acknowledgments}

This study was supported by the National Natural Science Foundation of China under Grants nos. 51579086, 51379068, and 51139001; Jiangsu Natural Science Foundation under Grant no. BK20140039; and Research Fund for the Doctoral Program of Higher Education of China under Grant no. 20120094110005.

\section{References}

[1] P. Rossi and F. Le Maou, "New method for detecting cracks in concrete using fibre optics," Materials and Structures, vol. 22, no. 6, pp. 437-442, 1989.

[2] F. Ansari and R. K. Navalurkar, "Kinematics of crack formation in cementitious composites by fiber optics," Journal of Engineering Mechanics, vol. 119, no. 5, pp. 1048-1061, 1993.

[3] H. Ohno, H. Naruse, M. Kihara, and A. Shimada, "Industrial applications of the BOTDR optical fiber strain sensor," Optical Fiber Technology, vol. 7, no. 1, pp. 45-64, 2001.

[4] C. K. Y. Leung, N. Olson, K. T. Wan, and A. Meng, "Theoretical modeling of signal loss versus crack opening for a novel crack sensor," Journal of Engineering Mechanics, vol. 131, no. 8, pp. 777790, 2005.

[5] K. T. Wan and C. K. Y. Leung, "Applications of a distributed fiber optic crack sensor for concrete structures," Sensors and Actuators, A: Physical, vol. 135, no. 2, pp. 458-464, 2007.

[6] L. Yuan, L. Zhou, and W. Jin, "Long-gauge length embedded fiber optic ultrasonic sensor for large-scale concrete structures," Optics \& Laser Technology, vol. 36, no. 1, pp. 11-17, 2004.

[7] Z. Zhang and F. Ansari, "Fiber-optic laser speckle-intensity crack sensor for embedment in concrete," Sensors and Actuators A: Physical, vol. 126, no. 1, pp. 107-111, 2006.

[8] K. S. C. Kuang, W. J. Cantwell, and C. Thomas, "Crack detection and vertical deflection monitoring in concrete beams using plastic optical fibre sensors," Measurement Science and Technology, vol. 14, no. 2, pp. 205-216, 2003.

[9] K. S. C. Kuang, S. T. Quek, and M. Maalej, "Assessment of an extrinsic polymer-based optical fibre sensor for structural health monitoring," Measurement Science and Technology, vol. 15, no. 10, pp. 2133-2141, 2004.

[10] K. S. C. Kuang, C. Y. Tan, S. H. Chew, and S. T. Quek, "Monitoring of large strains in submerged geotextile tubes using plastic optical fibre sensors," Sensors and Actuators A: Physical, vol. 167, no. 2, pp. 338-346, 2011.

[11] S. Liehr, P. Lenke, M. Wendt et al., "Polymer optical fiber sensors for distributed strain measurement and application in structural health monitoring," IEEE Sensors Journal, vol. 9, no. 11, pp. 13301338, 2009.

[12] S. Liehr, M. Wendt, and K. Krebber, "Distributed strain measurement in perfluorinated polymer optical fibres using optical frequency domain reflectometry," Measurement Science and Technology, vol. 21, no. 9, Article ID 094023, 2010.

[13] S. Liehr, N. Nöther, and K. Krebber, "Incoherent optical frequency domain reflectometry and distributed strain detection in polymer optical fibers," Measurement Science and Technology, vol. 21, no. 1, Article ID 017001, 2010.

[14] I. R. Husdi, K. Nakamura, and S. Ueha, "Sensing characteristics of plastic optical fibres measured by optical time-domain reflectometry," Measurement Science and Technology, vol. 15, no. 8, pp. 1553-1559, 2004.

[15] Y. B. Liao, Fiber Optics-Principles and Applications, Tsinghua University Press, Beijing, China, 1st edition, 2000 (Chinese). 


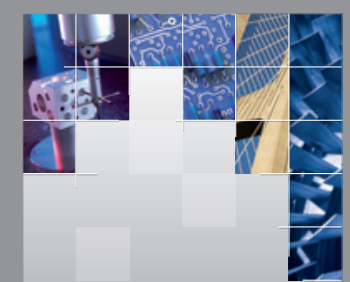

\section{Enfincering}
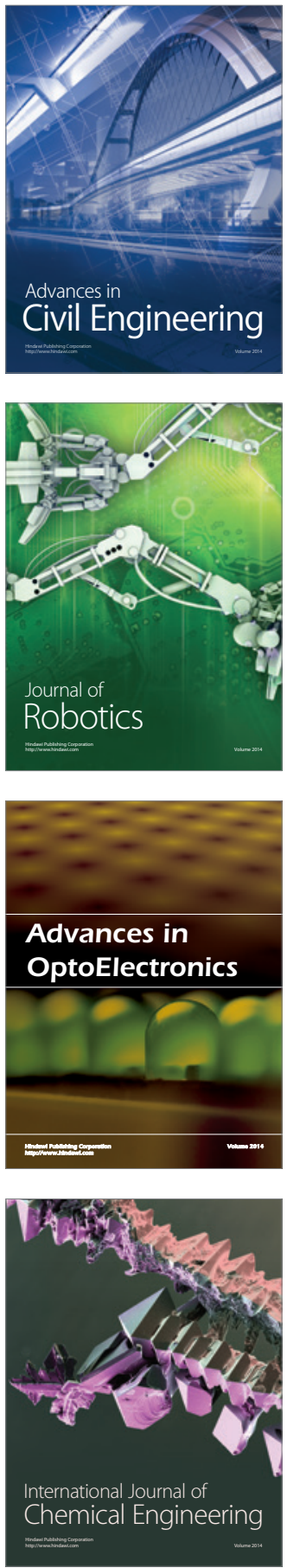

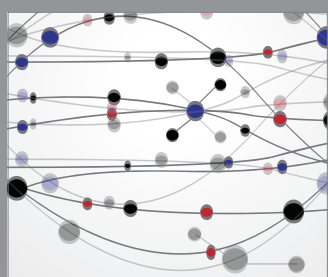

The Scientific World Journal

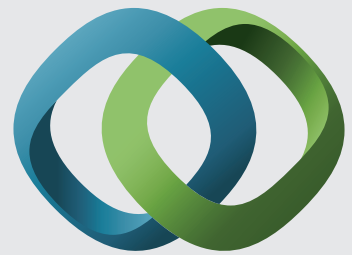

\section{Hindawi}

Submit your manuscripts at

http://www.hindawi.com
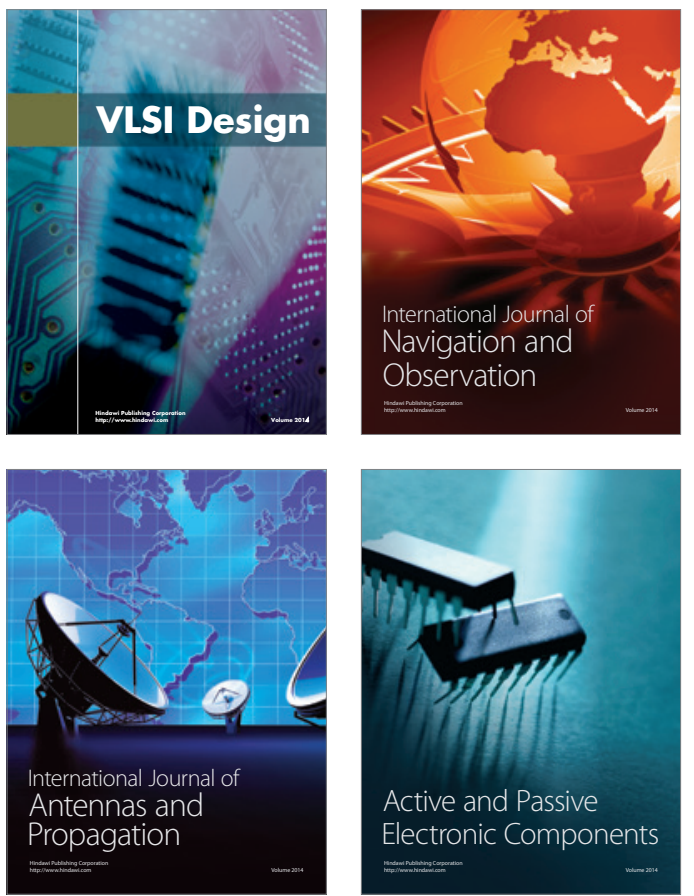
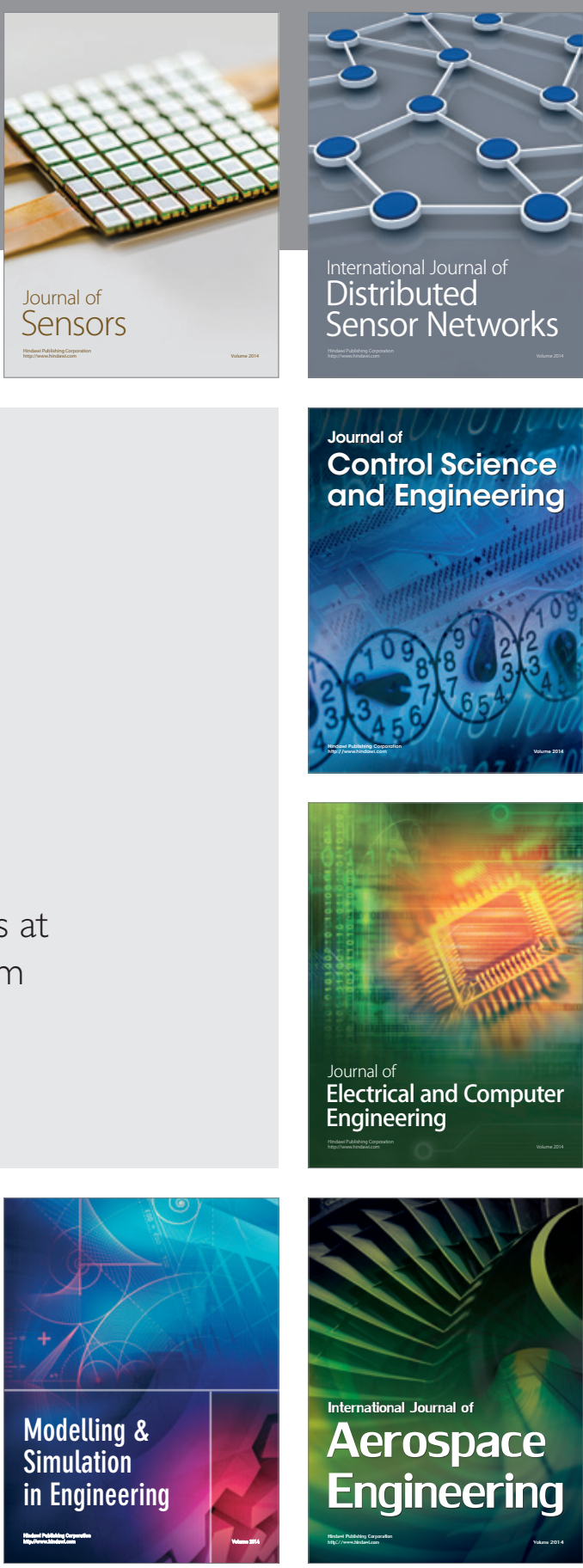

International Journal of

Distributed

Sensor Networks

Journal of

Control Science

and Engineering
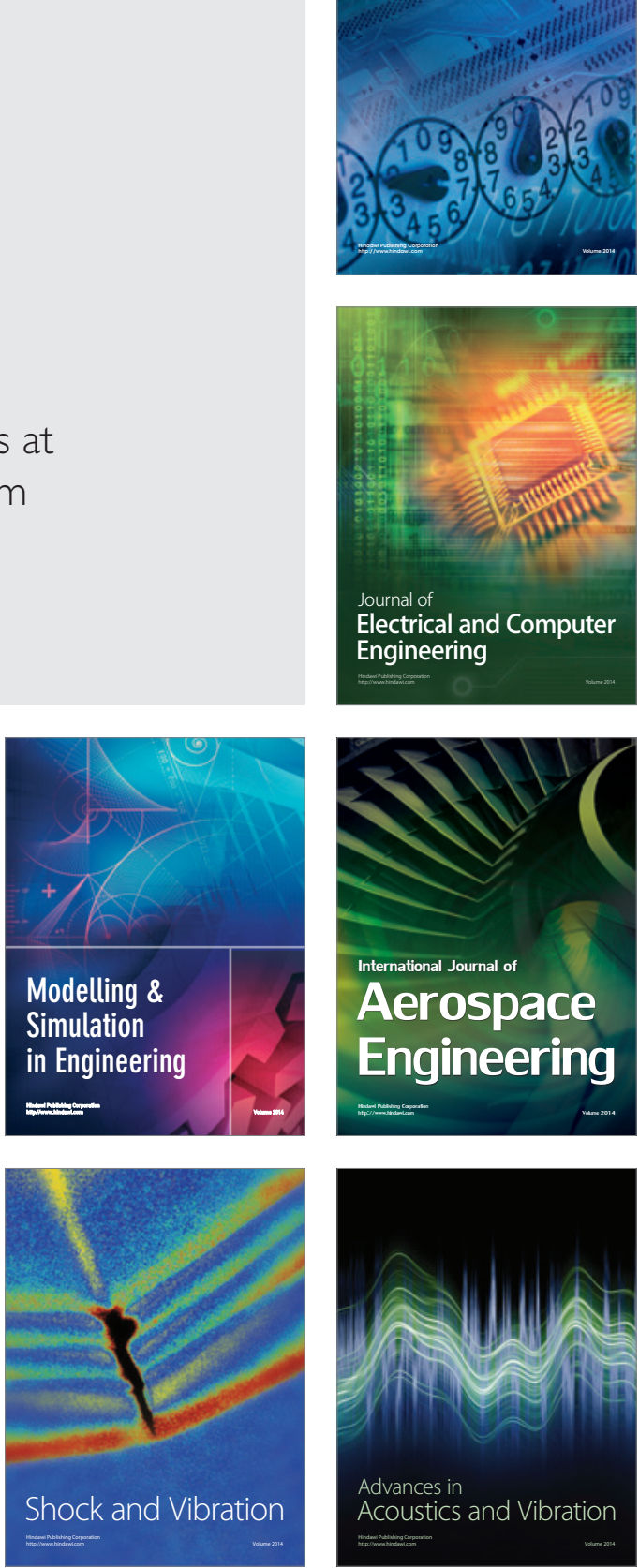